\title{
A Modified, Direct Neck Lift Technique: The Cervical Wave-Plasty
}

\author{
Fereydoun Don Parsa ${ }^{1}$, Nikki Castel ${ }^{2}$, Natalie Niloufar Parsa ${ }^{3}$ \\ ${ }^{1}$ Department of Surgery, Division of Plastic Surgery, University of Hawai'i, John A. Burns School of Medicine, Honolulu, HI; ${ }^{2}$ University of \\ Hawai'i, John A. BURNS School of Medicine, Honolulu, HI; ${ }^{3}$ Department of Family Practice, University of Hawai'i, John A. Burns School of \\ Medicine, Honolulu, HI, USA
}

Background Major problems with cervicoplasty by direct skin excision include the subjective nature of skin markings preoperatively and the confusing array of procedures offered. This technique incorporates curved incisions, resulting in a wave-like scar, which is why the procedure is called a "wave-plasty".

Methods This prospective study includes 37 patients who underwent wave-plasty procedures from 2004 to 2015. Skin pinching technique was used to mark the anterior neck preoperatively in a reproducible fashion. Intra-operatively, redundant skin was excised, along with excess fat when necessary, and closed to form a wave-shaped scar. Patients were asked to follow up at 1 week, 6 weeks, and 6 months after surgery.

Results The mean operation time was 70.8 minutes. The majority (81.3\%) was satisfied with their progress. On a scale of 1 to 10 ( 1 being the worst, and 10 being the best), the scars were objectively graded on average 5.5 when viewed from the front and 7.3 when seen from the side 6 months after surgery. Complications consisted of one partial wound dehiscence (2.3\%), one incidence of hypertrophic scarring (2.3\%), and two cases of under-resection requiring revision (5.4\%).

Conclusions In select patients, surgical rejuvenation of the neck may be obtained through wave-like incisions to remove redundant cervical skin when other options are not available. The technique is reproducible, easily teachable and carries low morbidity and high patient satisfaction in carefully chosen patients.

Keywords Cervicoplasty / Neck / Rejuvenation

\section{Correspondence:}

Fereydoun Don Parsa

Department of Surgery, Division of

Plastic Surgery, University of Hawai'i,

John A. Burns School of Medicine,

1329 Lusitania Street, Suite 807,

Honolulu, HI 96813, USA

Tel: +1-808-526-0303

Fax: +1-808-536-8836

E-mail: fdparsa@gmail.com
No potential conflict of interest relevant to this article was reported.

\section{INTRODUCTION}

A youthful neck is characterized by a distinct inferior mandibular border, visible subhyoid depression, visible thyroid cartilage bulge, anterior sternocleidomastoid line at a 90-degree angle with the submental line, and cervical angle of 105 to 120 degrees. However, as people age, the neck loses its natural shape and contour due to fat accumulation in the submental region, banding of the platysmal muscle, and anterior cervical skin laxity $[1-8]$.

Neck rejuvenation is achievable through several different surgical options. A full face and neck lift results in the best improvement and has well concealed periauricular scars. However, an increasing number of patients, in particular the male and older 


\section{Fig. 1. Various skin excisional techniques}

Top left figure is a vertical elliptical incision described in 1903 with a simple closure. Top right is a Z-incision from 1964 (Marino) with a Z-plasty closure. Bottom left figure shows multi-Z-incisions (1971, Cronin and Biggs) with multi-Z-plasties. Bottom right figure is the current wave-plasty technique with wave-like incisions and simple closure.

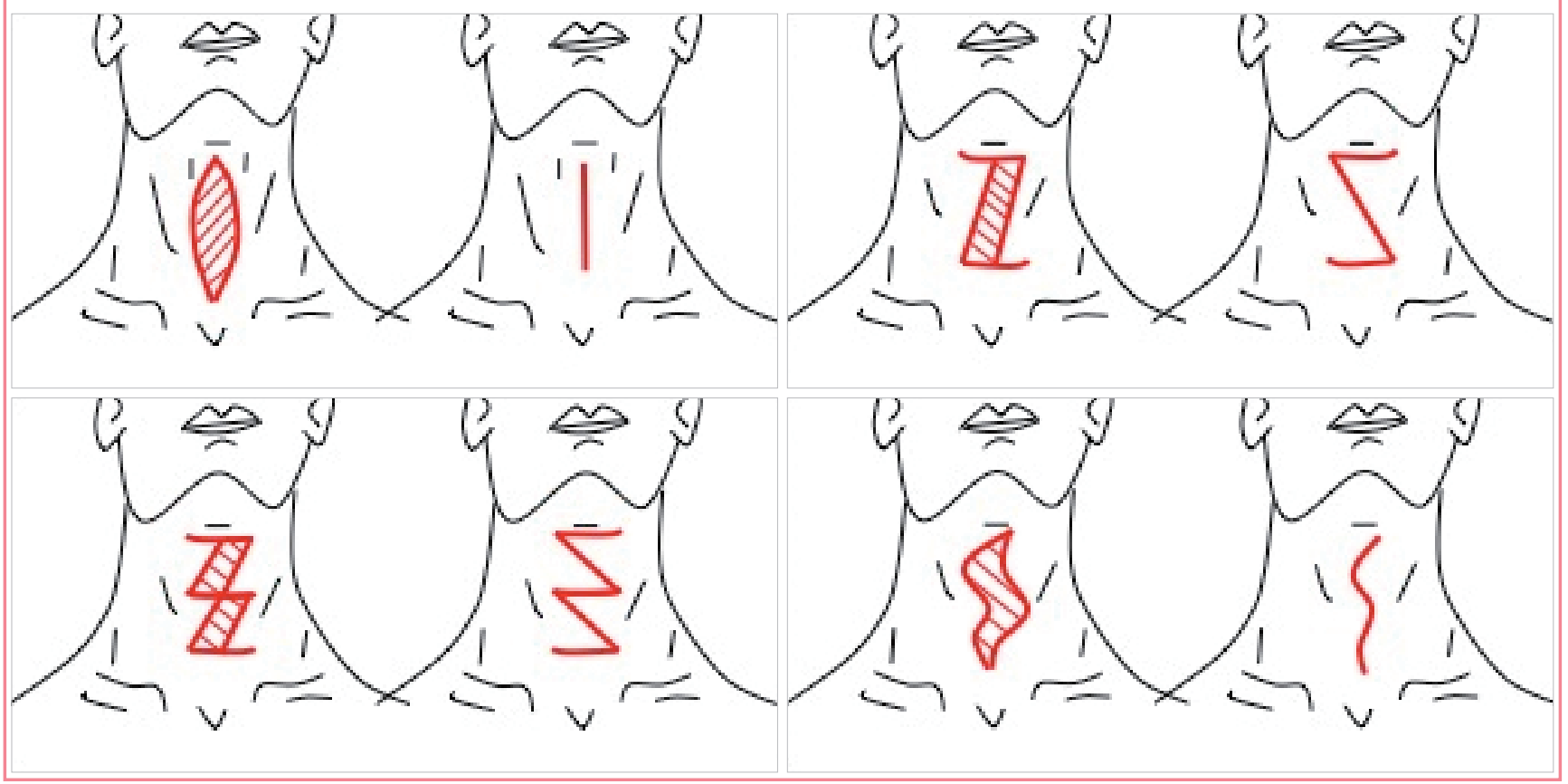

\section{Fig. 2. Preoperative markings completed}

Initially, mark a horizontal submental line, measuring about $4 \mathrm{~cm}$, with point $X$ at the left end of the line. Then draw a vertical midline, approximately $14-16 \mathrm{~cm}$, from the midpoint of the submental line to the sternal notch (marked point Y). Divide the midline into four equal segments, by making three division markings. Mark points $A, B, C$, $D, E$, and $F$ using the pinching technique described in Fig. 3. Connect the points to draw the curve $X-A-C-E-Y$. Draw curve $X-$ B-D-F-Y after further pinching the skin with forceps, in approximately one-centimeter intervals, to assure that the entire length of the wound can be closed under no tension. The left shows a Type I marking requiring Type I closure. The right shows a Type II or Type III marking, where X-A-C is significantly longer than $X-B-D$, requiring Type II or III excision as shown in Fig. 4.

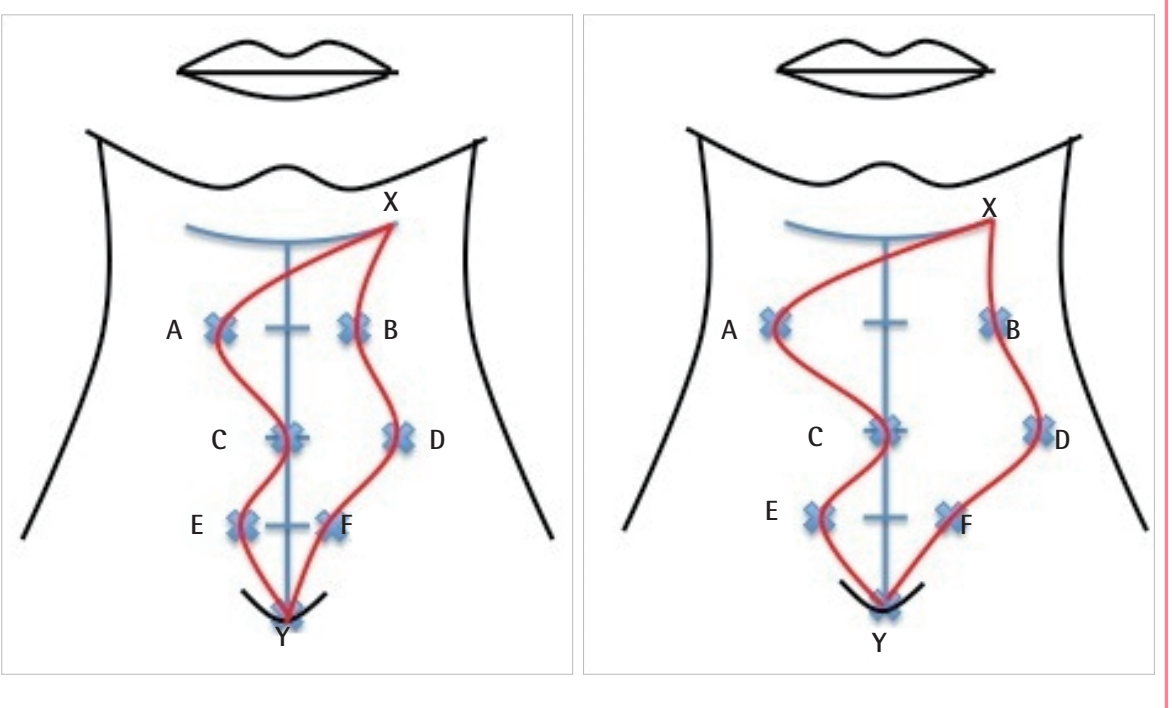

population, are only interested in improving their neck contour and prefer a shorter, less extensive, and less costly procedure or are not suitable for longer operations due to medical reasons.

There is a wide array of procedures for neck rejuvenation through direct skin excision [1-7]. The major approaches are summarized in Fig. 1. These procedures may include fat removal and platysma plication. A major problem with these approaches is the relatively subjective nature of skin markings prior to surgery
[1-7]. The senior author (F.D.P.) has tried many of the available techniques over the past 35 years. Lacking full satisfaction with these methods, he designed the present prospective study in 2004 to offer a simple, easily teachable, and reproducible technique with precise preoperative markings. An overview of this technique is shown in Figs. 2 and 3.

This technique incorporates curved incisions, resulting in a final wave-like scar, which is why we refer to this procedure as a cervi- 


\section{Fig. 3. Preoperative markings for the wave-plasty}

(A) Step 1: Grab redundant cervical skin with smooth tip forceps at the level of the first dividing mark, with tips equidistant from the midline on each side. The skin at the tips of the forceps is marked as points A and B. (B) Step 2: Using the second dividing mark on the midline, mark points $\mathrm{C}$ and $\mathrm{D}$ by grabbing the excess skin on the patient's left side by placing one forceps tip on the midline and the other on the left side of the neck. (C) Step 3: Mark points E and F by again grabbing excess cervical skin with forceps tips equidistant from vertical midline at the level of the last division mark.

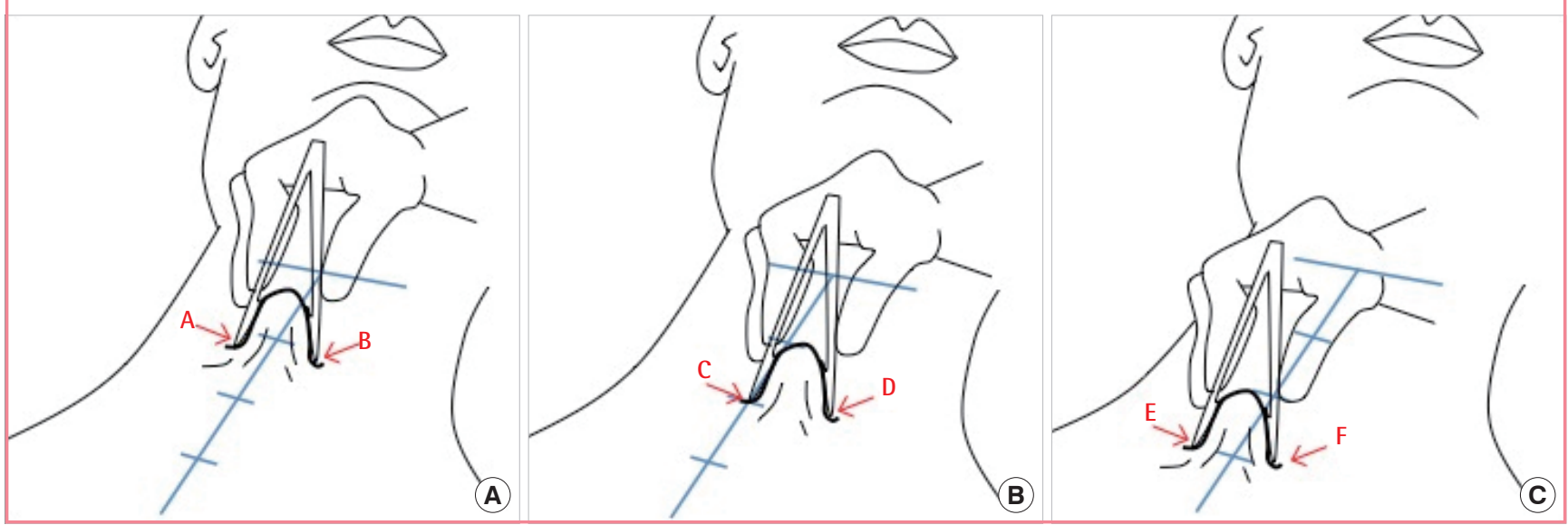

\section{Table 1. Patient demographics}

\begin{tabular}{|lc|}
\hline Characteristic & No. of patients (\% of total) \\
\hline Age (yr) & \\
Range & $43-75$ \\
Average & 60 \\
Race & \\
Caucasian & $20(54.1)$ \\
Asian & $8(21.6)$ \\
Mixed Caucasian/Asian & $9(24.3)$ \\
Sex & \\
Male & $16(43.2)$ \\
Female & $21(56.8)$ \\
Previous face lift & $14(37.8)$ \\
Comorbidity & \\
Smoking & $8(21.6)$ \\
Hypertension & $8(21.6)$ \\
Obesity (body mass index $\geq 30)$ & $7(18.9)$ \\
Diabetes mellitus type 2 & $2(5.4)$ \\
Rheumatoid arthritis & $2(5.4)$ \\
History of myocardial infarction & $2(5.4)$ \\
\hline
\end{tabular}

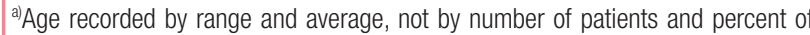
total.

cal "wave-plasty” (Fig. 2). Curved incisions result in better healing and are less likely to result in hypertrophic or keloid scars [7].

\section{METHODS}

\section{Patient selection}

Between July 2004 and February 2015, the cervical wave-plasty was performed on 37 patients (Table 1). The patient was selected for this operation if the primary complaint was cervical skin laxity, he or she would not accept a standard face and neck lift, and he or she clearly understood the location of the resulting

\section{Table 2. Scar grading}

\begin{tabular}{|lcc|}
\hline Quality of scar & Grade & Width or height of scars $(\mathrm{mm})$ \\
\hline Poor & 1 & $>3.0$ \\
Poor to acceptable & 2 & $>2.6$ \\
& 3 & $>2.3$ \\
& 4 & $>2.0$ \\
Acceptable to good & 5 & $>1.6$ \\
& 6 & $>1.3$ \\
Good & 7 & $>0.6$ \\
& 8 & $>0.3$ \\
& 9 & $\leq 0.3$ \\
\hline
\end{tabular}

scars on the neck and the potential for developing widened, hypertrophic, or keloid scarring.

Patients were informed that both the patient and the physician would score their resulting scar six months after surgery based on an objective system (Table 2). The operation was not scheduled unless patients clearly understood that the resulting scars would always remain visible, with the potential for developing unsightly scars.

\section{Operative technique}

The anterior neck was marked while the patient was seated or standing (Figs. 2, 3). A horizontal submental mark was drawn with Point $\mathrm{X}$ on the left. A vertical midline mark was drawn down to the sternal notch (Point $\mathrm{Y}$ ) and divided into four parts using three division markings. Excess cervical skin was pinched at each division marking to determine points along the curves (Fig. 3). The points were connected to draw 2 curves: X-A-C-E-Y and XB-D-F-Y. The latter curve was drawn after the skin was again pin- 


\section{Fig. 4. Variations of closure}

Image on the left shows a Type I closure (56.8\% of patients) where X-A-C-E-Y and X-B-D-F-Y were simply approximated. Image in the middle is a Type II closure with dog-ear excision from within the submental area (29.7\% of patients). Image on the right is a Type III closure. Because $X-C$ is much longer than X-D, additional skin was excised horizontally at the level of the first division in the upper neck in order to close the incision under no tension (13.5\% of patients).
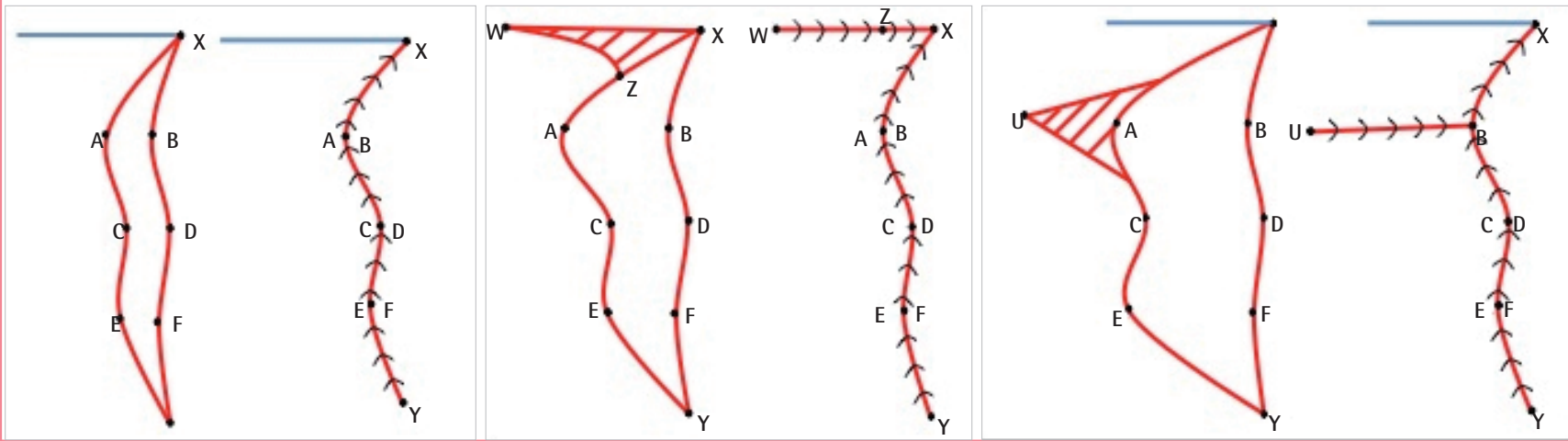

Fig. 5. Preoperative to postoperative photographs

A 72-year-old male patient (A) with laxity of cervical skin preoperatively in the front view, (B) laxity preoperatively in the side view, (C) after Type I skin closure, and two months after surgery from the (D) front view, and (E) side view.
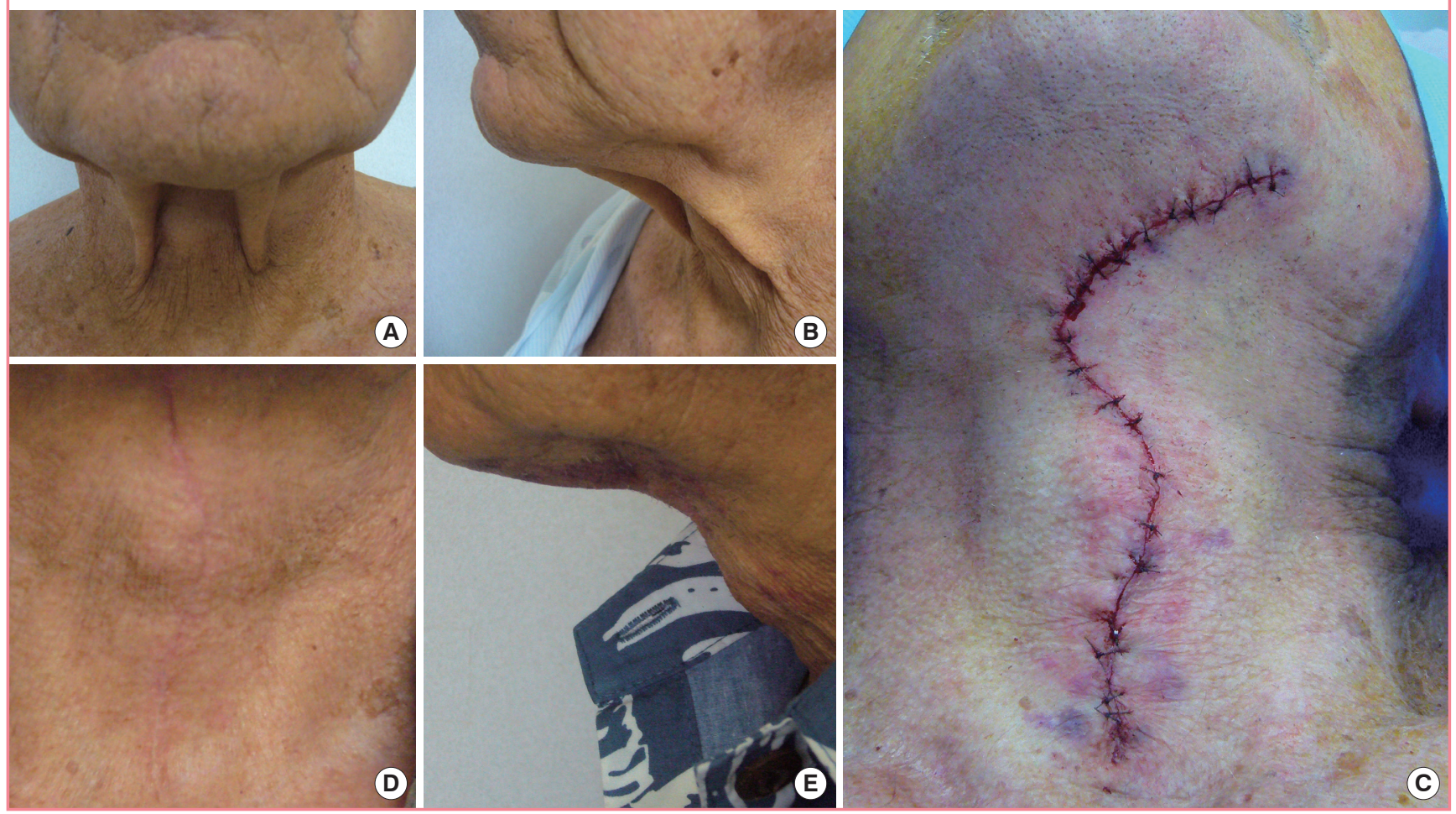

ched with forceps to assure that the entire length of the wound would be closed under no tension. The senior surgeon (F.D.P.) performed all procedures as an outpatient under light intravenous sedation and local anesthesia. Incisions were made along the two wave-like curves joining point $\mathrm{X}$ in the submental area to point $\mathrm{Y}$ in the sternal notch (Figs. 2, 3). Redundant skin was excised and the platysmal muscles were plicated in the midline with interrupted 4-0 absorbable monofilament sutures. Lipec- tomy was performed if there was excess subcutaneous, submental and subplatysmal fatty deposits.

One of three types of final wound closures were performed depending on the extent of skin redundancy (Fig. 4). Type I involved a simple closure. Type II excised any dog ear in the submental area. Type III excised skin horizontally before closing the incision in order to minimize tension.

Skin margins were approximated with interrupted 4-0 absorb- 
Table 3. Operative length and 6-month postoperative results

\begin{tabular}{|lc|}
\hline Procedure outcome & Range (average) \\
\hline Length of operation (min) & \\
All surgeries & $40-110(70.8)$ \\
No SML & $40-100(66.7)$ \\
With SML & $75-110(91.7)$ \\
Grades of the scars at 6 months & \\
Viewed from front (average grade) & 5.5 \\
Viewed from side (average grade) & 7.3 \\
Satisfaction rate at 6 months (\%) & 81.3 \\
\hline SML, submental lipectomy. & \\
\hline
\end{tabular}

able monofilament intradermal sutures and closed with external 5-0 nylon interrupted sutures (Fig. 5C). Finally, wounds were covered with 1-inch micropore paper tape and patients were instructed to continue paper tape application for 6 months while at home.

\section{RESULTS}

Cervical wave-plasty was performed on 37 patients. Demographics are summarized in Table 1. Patients ranged in age from 43 to 75 years old with an average of 60 years. Although women were the predominant gender (56.8\%), there was a significant male population (43.2\%). More than half $(51.4 \%)$ of the patients had pre-existing comorbidities, most notably smoking (21.6\%), hypertension (21.6\%) and obesity with body mass index of 30 or higher $(18.9 \%)$.

The majority of patients (86.5\%) underwent either Type I (Fig. 4A) or Type II skin closures (Fig. 4B). The operation ranged from 40 to 110 minutes (average 70.8 minutes) from the time of incision to the completion of closure. Operations including submental lipectomy ranged from 75 to 110 minutes (average, 91.7 minutes), while operations without submental lipectomy ranged from 40 to 100 minutes (average, 66.7 minutes), as shown in Table 3.

Postoperatively, more than half of the patients (53\%) did not require any analgesics, none required narcotics, and all were able to return to limited routine daily activities the day after surgery $[9,10]$. Thirty-two patients $(86 \%)$ returned for the 6-month follow-up visit. Before and after photographs are shown in Figs. 5 and 6.

Complications included four patients ( $12.5 \%$ of patients) who presented with partial recurrent laxity of the cervical skin. Two of the four (6.2\%) underwent revisions at 6 and 11 months after the initial operation with satisfactory outcome. A 71-year-old former smoker experienced a $1.5 \mathrm{~cm} \times 1.5 \mathrm{~cm}$ wound dehiscence in the upper neck. The area healed uneventfully in 3 weeks. A 72- year-old male developed a $2 \mathrm{~cm}$ long hypertrophic scar in
Table 4. Complications

\begin{tabular}{|lc|}
\hline Complication & No. of patients (\%) \\
\hline Dehiscence & $1(3.1)$ \\
Hypertrophic scar & $1(3.1)$ \\
Recurrence of laxity & $4(12.5)$ \\
Requiring revision & $2(6.2)$ \\
Not requiring revision & $2(6.2)$ \\
Hematoma & 0 \\
Infection & 0 \\
\hline
\end{tabular}

Table 5. Anonymous questionnaire responses one year after surgery

\begin{tabular}{|lr|}
\hline Response & No. (\%) \\
\hline No. of responses one year after surgery $(n=37)$ & $32(86.5)$ \\
Responders with complaints $(n=32)$ & $4(12.5)$ \\
Responders who were satisfied & $32(100)$ \\
Responders who would do it again & $32(100)$ \\
Rating of scar from the front view $(n=32)$, range (average) & $6-9(7.68)$ \\
Rating of scar from the side view $(n=32)$, range (average) & $8-10(9.71)$ \\
\hline
\end{tabular}

the submental area. Although the area of hypertrophy was limited, this scar received the grade of 1 . There were no other complications (Table 4).

Six months after surgery, the scars received an average grade of 5.5 when viewed from the front, and 7.3 when from the side view (Table 3 ). These grades translate into scars classified as "acceptable to good" in the grading system utilized (Table 2). Six patients were unsatisfied with their results: the patient with dehiscence, the patient with hypertrophic scarring, and the four patients with recurrent laxity.

The responses from the questionnaire in Fig. 7 are shown in Table 5. Of the 32 responders, 28 patients (87.5\%) had no complaints, all (100\%) would do the procedure again, and all (100\%) would recommend the procedure to others. The four patients who were not satisfied with the procedure may have been part of the six patients previously unsatisfied at the six-month followup visit, however this cannot be known for certain as it was an anonymous survey.

Few patients have returned following the six-month follow-up period, other than for complications mentioned above. Some patients have returned to the office for other purposes. They have maintained their good results up to five years after surgery, as shown in Fig. 6E and F.

\section{DISCUSSION}

Although women made up at least $90 \%$ of the patient population in four different rhytidectomy studies [11-13], this study is 
Fig. 6. Preoperative and postoperative photos of 65 -year-old female

A 65-year-old female. (A) Preoperative front view. (B) Preoperative side view. (C) 6-month postoperative front view. (D) 6-month postoperative side view. (E) 5-year postoperative front view. (F) 5-year postoperative side view.
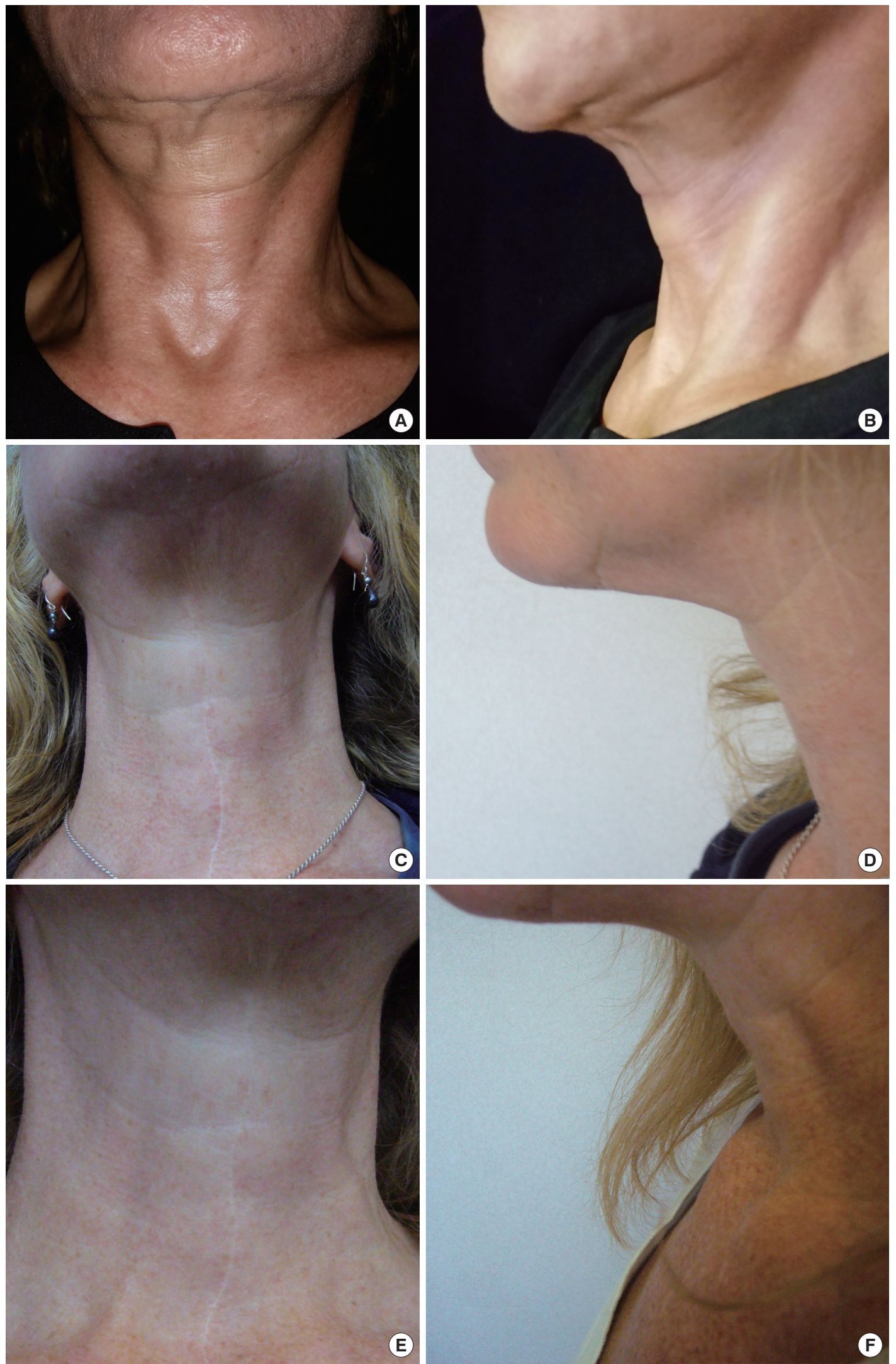


\section{Fig. 7. Anonymous questionnaire}

Anonymous questionnaire mailed to all patients one year after surgery.

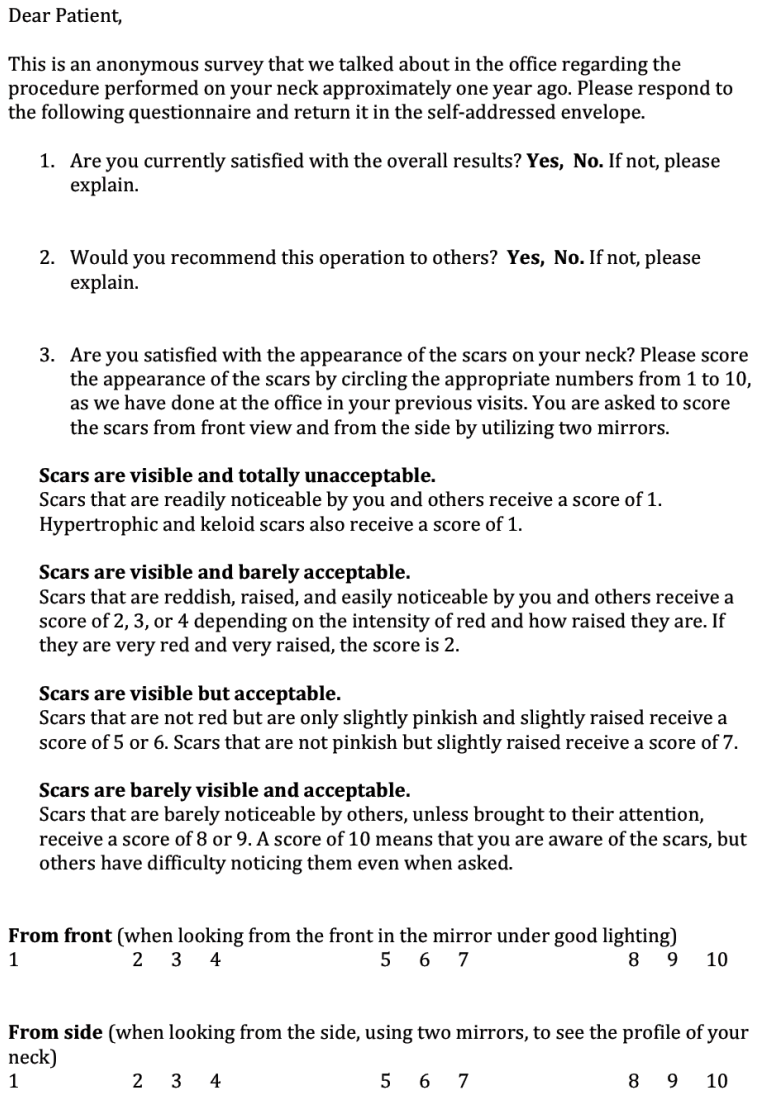

much more representative of male patients. This finding suggests that men in this study were primarily concerned with the appearance of their neck and were quite receptive to scars as a tradeoff with improved neck contour.

Over half of the patients (51.4\%) had at least one pre-existing comorbidity (Table 1). Obesity was a frequent comorbidity (18.9\%). Ideally patients undergoing neck lifts have thin necks with excess cervical skin and minimal subcutaneous fat. These patients achieve aesthetically pleasing neck contour with the excision of the excess skin. However, obese patients have excess subcutaneous fat in the neck are more difficult to contour.

Over $40 \%$ gave a history of smoking, hypertension, or both. Smokers, including former smokers, have a higher risk of postoperative complications $[14,15]$. This higher incidence in this study is likely related to patient selection. Both smokers and former smokers were informed that they carry a higher complication rate, which likely influenced them to choose this less invasive procedure.

Despite the fact that age, obesity, diabetes, hypertension and smoking are important comorbid factors in wound healing [15] we encountered only one case (3.1\%) of wound dehiscence that occurred in a former smoker (Table 4). Hypertension is another important comorbidity because of the potential for postoperative hematoma [16]. In this study, $21.6 \%$ of patients had hypertension. However we had no incidence of hematoma. Wave-like incisions, minimal to no undermining, and tension-free skin closure were essential in minimizing wound complications. Platysma muscle plication was likely helpful in both reducing tension on wound margins and improving neck contour. The use of micropore paper tape over the incisions for 6 months is also helpful in improving the appearance of the scars [17-19].

Six months after surgery the scars received an average grade of 5.5 from the front and 7.3 from the side (Table 3 ). The grades were based on the worst sections of the scars. Most of the scar was barely visible from the side view (Fig. 6D). This study was limited to 6 months of follow-up and further improvement of scars after this period is usually expected [17]. Subjectively, $81.3 \%$ of patients at the six-month follow-up visit and $87.5 \%$ of respondents to the one-year anonymous survey were completely satisfied with both their scars and their neck contour and would recommend the procedure to others. Because the one-year survey was anonymous, it is unknown whether the 32 responders to the one-year survey were the same 32 patients who returned for the six-month follow-up visit. There was one incidence of hypertrophic scarring in a 72-year-old male who had no complaints and did not want any treatment. Previous studies of standard face and neck lift have reported an incidence of hypertrophic scarring from less than $2 \%$ to as high as $18 \%[13,20-22]$.

Four patients (12.5\%) showed partial recurrence of laxity six months after surgery. The recurrent laxity was minor, with return of no more than $10 \%$ of the original cervical skin laxity. Patients should be warned prior to surgery that some return of laxity might occur with time, comparable to a standard face and neck lift procedure. To minimize this recurrence, optimal amount of skin should be excised without putting undue tension on the closure.

In conclusion, this prospective study describes an innovative, simple, and reproducible approach to neck rejuvenation utilizing curvilinear incisions. It allows for accurate preoperative planning and markings, results in a pleasing wave-like scar, and allows for quick return to daily activities with minimal need for postoperative pain medication. This procedure appears most suitable for older patients who clearly understand and accept the resulting scars as a trade-off for an improved neck contour.

\section{REFERENCES}

1. Rohrich RJ, Rios JL, Smith PD, et al. Neck rejuvenation re- 
visited. Plast Reconstr Surg 2006;118:1251-63.

2. Ramirez OM. Cervicoplasty: nonexcisional anterior approach: a 10-year follow-up. Plast Reconstr Surg 2003;111:1342-5.

3. Miller TA. Excision of redundant neck tissue in men with platysma plication and Z-plasty closure. Plast Reconstr Surg 2005;115:304-13.

4. Chaput B, Grolleau JL, De Bonnecaze G, et al. Anterior medial cervicoplasty. Ann Chir Plast Esthet 2012;57:612-7.

5. Bitner JB, Friedman O, Farrior RT, et al. Direct submentoplasty for neck rejuvenation. Arch Facial Plast Surg 2007;9: 194-200.

6. Bruneau S, Nguyen P, Foletti JM, et al. Neck rejuvenation by anterior cervicoplasty: an original technical modification. J Plast Reconstr Aesthet Surg 2013;66:1627-8.

7. Jordan JR, Yellin S. Direct cervicoplasty. Facial Plast Surg 2014;30:451-61.

8. Sugai DY, Deptula PL, Parsa AA, et al. The importance of communication in the management of postoperative pain. Hawaii J Med Public Health 2013;72:180-4.

9. Parsa AA, Sprouse-Blum AS, Jackowe DJ, et al. Combined preoperative use of celecoxib and gabapentin in the management of postoperative pain. Aesthetic Plast Surg 2009;33: 98-103.

10. Parsa AA, Soon CW, Parsa FD. The use of celecoxib for reduction of pain after subpectoral breast augmentation. Aesthetic Plast Surg 2005;29:441-4.

11. Conway H. The surgical face lift--rhytidectomy. Plast Reconstr Surg 1970;45:124-30.

12. Kamer FM, Frankel AS. Isolated submentoplasty: a limited approach to the aging neck. Arch Otolaryngol Head Neck Surg 1997;123:66-70.

13. Friel MT, Shaw RE, Trovato MJ, et al. The measure of face- lift patient satisfaction: the Owsley Facelift Satisfaction Survey with a long-term follow-up study. Plast Reconstr Surg 2010;126:245-57.

14. Riefkohl R, Wolfe JA, Cox EB, et al. Association between cutaneous occlusive vascular disease, cigarette smoking, and skin slough after rhytidectomy. Plast Reconstr Surg 1986; 77:592-5.

15. Parsa AA, Nakasone GK, Soon CW, et al. Smoking and breast reduction. Plast Reconstr Surg 2006; 117:2506-7.

16. Baker DC, Stefani WA, Chiu ES. Reducing the incidence of hematoma requiring surgical evacuation following male rhytidectomy: a 30-year review of 985 cases. Plast Reconstr Surg 2005; 116:1973-85.

17. Parsa FD, Parsa NN, Murariu D. Surgical correction of the frowning mouth. Plast Reconstr Surg 2010;125:667-76.

18. Reiffel RS. Prevention of hypertrophic scars by long-term paper tape application. Plast Reconstr Surg 1995;96:1715-8.

19. Atkinson JA, McKenna KT, Barnett AG, et al. A randomized, controlled trial to determine the efficacy of paper tape in preventing hypertrophic scar formation in surgical incisions that traverse Langer's skin tension lines. Plast Reconstr Surg 2005; 116:1648-56.

20. Henderson J, O’Neill T, Logan A. Direct anterior neck skin excision for cervicomental laxity. Aesthetic Plast Surg 2010; 34:299-305.

21. Matarasso A, Elkwood A, Rankin M, et al. National plastic surgery survey: face lift techniques and complications. Plast Reconstr Surg 2000;106:1185-95.

22. Thompson DP, Ashley FL. Face-lift complications: a study of 922 cases performed in a 6-year period. Plast Reconstr Surg 1978;61:40-9. 